\title{
Kelly Starret mit Juliet Starret und Glen Cordoza: Sitzen ist das neue Rauchen. Das Trainingsprogramm, um Haltungsschäden vorzubeugen und unsere natürliche Mobilität zurückzugewinnen. Riva Verlag: München 2016. ISBN: 978-3-86883-800-8
}

Im Rahmen der CSR-Arbeitsgruppe an der UB Wien werden in regelmäßigen Abständen Bücher ausgewählt und vorgestellt, die thematisch zu unseren Arbeitsschwerpunkten passen und allen Leserinnen und Lesern Lust darauf machen sollen, sich mit spannenden, aktuellen Themen auseinanderzusetzen. Alle präsentierten Bücher sind an der Universitätsbibliothek verfügbar.

„Sitzen ist das neue Rauchen“, das ist ein Buchtitel, der sicherlich Reaktionen und möglicherweise auch Emotionen weckt, auf jeden Fall macht er neugierig und ist eine gute Einstimmung auf

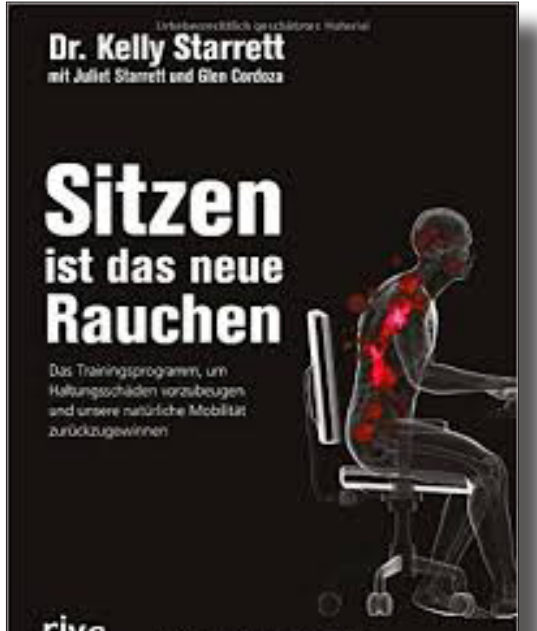

riva das, was einen in diesem Buch erwartet. Das sanfte „schön reden“ von unangenehmen Tatsachen ist nicht die Sache des Autors Kelly Starret vor den negativen Auswirkungen unseres sitzenden Lebensstils wird in sehr eindringlicher Form gewarnt. Wir sitzen beim Frühstück, am Weg in die Arbeit, in der Arbeit, zu Hause vor dem Computer und vor dem Fernseher. Dabei schalten die Muskeln der unteren Körperzonen ab und wir nehmen Haltungen ein, bei denen die Muskeln, die zum Stützen von Wirbelsäule und Rumpf wichtig sind, nicht aktiviert werden. Daraus ergeben sich geschwächte Körperfunktionen, die viele orthopädische Probleme mit sich ziehen und die auch durch sportliches Freizeitprogramm nicht ausgeglichen werden können. Nur sehr wenige Menschen, die einer überwiegend sitzenden Tätigkeit nachgehen, sind komplett schmerzfrei.

Der Autor und sein Team liefern aber nicht nur unangenehme Fakten, sondern sie bieten auch konkrete Lösungsansätze dafür an, wie man mehr Bewegung in den Alltag bringen kann. Die wenigsten Leserlnnen werden 
die Möglichkeit haben, sich im Büro einen Steharbeitsplatz einzurichten, wie es im Buch empfohlen wird, aber vielleicht ist es möglich, die eine oder andere Besprechung im Stehen abzuhalten, stehend zu telefonieren oder immer wieder aufzustehen und kleine Bewegungseinheiten einzulegen. So tut man nicht nur etwas Gutes für seinen Körper, sondern es profitieren auch die Konzentrationsfähigkeit und die Produktivität davon. Das Buch bietet außerdem Mobilisationspläne für alle Körperregionen und man kann, lernen wie man mit Grundlagen-Körperarbeit Schmerzen beseitigen und die Beweglichkeit steigern kann.

Wem es zu viel ist, sich durch das mit 362 Seiten doch sehr umfangreiche Werk zu lesen, dem sei zumindest das Kapitel „Überlebensstrategien im Sitzen“ ans Herz gelegt, das mit vielen praktischen Tipps gefüllt ist, die sich in den Büroalltag übernehmen lassen.

Wer sich auf dieses Buch einlässt, den wird es möglicherweise im wahrsten Sinne des Wortes vom Hocker reißen, denn es ist eigentlich unmöglich, dieses Buch im Sitzen zu lesen.

\section{Mag. ${ }^{a}$ Anna Krenn Universität Wien, Bibliotheks- und Archivwesen Universitätsring 1, A-1010 Wien E-Mail: anna.krenn@univie.ac.at}

Dieses Werk ist lizenziert unter einer

Creative-Commons-Lizenz Namensnennung 4.0 International 